\title{
TTR
}

Traduction, terminologie, re?daction

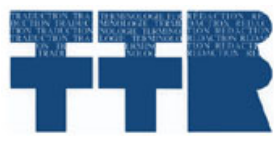

\section{Les voix parallèles de Nancy Huston}

\section{Christine Klein-Lataud}

Volume 9, numéro 1, 1er semestre 1996

Le festin de Babel

Babel's Feast

URI : https://id.erudit.org/iderudit/037245ar

DOI : https://doi.org/10.7202/037245ar

Aller au sommaire du numéro

\section{Éditeur(s)}

Association canadienne de traductologie

ISSN

0835-8443 (imprimé)

1708-2188 (numérique)

Découvrir la revue

Citer cet article

Klein-Lataud, C. (1996). Les voix parallèles de Nancy Huston. TTR, 9(1), 211-231. https://doi.org/10.7202/037245ar

\section{Résumé de l'article}

Les voix parallèles de Nancy Huston - Cet article s'attache à la problématique des écrivains bilingues ou polyglottes (Eisa Triolet, Vladimir Nabokov, Julien Green), et plus particulièrement à celle de Nancy Huston. Quelles raisons poussent un écrivain ou une écrivaine à utiliser pour la création une langue seconde plutôt que sa langue maternelle ? Quand l'auteur s'auto-traduit, s'agit-il d'une nouvelle création ? Nancy Huston était-elle en droit de recevoir le Prix du gouverneur général dans la catégorie fiction fancophone pour Cantique des plaines, « réécriture » de Plainsong par l'auteure?
Tous droits réservés @ C TTR: traduction, terminologie, rédaction — Les auteurs, 1996
Ce document est protégé par la loi sur le droit d'auteur. L'utilisation des services d'Érudit (y compris la reproduction) est assujettie à sa politique d'utilisation que vous pouvez consulter en ligne.

https://apropos.erudit.org/fr/usagers/politique-dutilisation/ 


\section{Les voix parallèles de Nancy Huston}

\section{Christine Klein-Lataud}

Ne pas oublier les oiseaux. Le rossignol ne chantera ni cou-cou ni cra-cra, le corbeau n'émettra pas de trilles. Jamais. Qu'ils sortent de l'cuf ici ou là, le langage des oiseaux est, comme le plumage, attaché à l'espèce. [...] Pour l'homme, il existe une langue maternelle, son premier mode d'expression ; ensuite, il peut l'oublier, en apprendre une ou plusieurs autres sans oublier la première. L'homme est capable de s'exprimer dans les cou-cou, cra-cra et autres trilles humains. (Elsa Triolet, 1969 , p. 7)

C'est ainsi que s'ouvre la Mise en mots, texte où, à la fin de sa vie, Elsa Triolet raconte ses rapports avec l'écriture. L'homme y est défini non comme l'animal doué de langage, mais comme l'étrange animal capable d'en utiliser plusieurs. Le seul animal, donc, confronté au problème du choix.

Cet article s'attache à la problématique des écrivains bilingues ou polyglottes, et plus particulièrement à Nancy Huston.

Rappelons tout d'abord que le bilinguisme a été et reste de règle dans bien des pays et qu'il le fut longtemps en Europe, en particulier au Moyen Âge, époque où, quelle que fût sa langue vernaculaire, on écrivait en latin. Mais souvent il s'agit d'un bilinguisme assorti de spécialisation fonctionnelle, une langue étant 
réservée aux échanges quotidiens, l'autre à l'étude et à la création. Je ne m'intéresserai ici qu'aux rapports des écrivains avec la ou les langues qu'ils ont utilisées comme moyen d'expression et de création, à leur navigation spectaculaire et souvent dramatique entre langue maternelle et langue d'adoption.

Qu'est-ce qui pousse un écrivain à utiliser pour la création une langue autre que sa langue maternelle ? Ce sont parfois les aléas de l'histoire, histoire personnelle ou " Histoire avec une grande Hache ", comme disait Georges Perec. Contraint à l'exil, l'écrivain finit par adopter la langue du pays d'accueil, pour des raisons d'ordre pratique (contraintes éditoriales, nécessité de se trouver un public) ou pour des motifs plus personnels (adaptation au nouvel environnement linguistique et culturel).

Cependant, l'exil ou l'expatriation ne suffisent pas à susciter un changement de langue, comme le prouve l'exemple de nombreux auteurs qui, tels Maria Tsvetaîeva, n'ont écrit que dans leur langue maternelle, quoiqu'ils aient parfaitement maîtrisé la langue de leur nouveau pays de résidence. L'adoption d'une autre langue est un choix, comme le souligne Jorge Semprun. Opposant son cas à celui d'autres Espagnols exilés en France qui ont " délibérément limité leur usage correct du français à des fins utilitaires », il explique que, pour sa part, il a décidé de choisir comme langue de création « le français, langue de l'exil » et qu'il l'a transformé en " une autre langue maternelle, originaire ". Cette démarche lui a permis de se créer une patrie coupée du " terreau matriciel " de la langue originaire et de se constituer linguistiquement et fantasmatiquement de nouvelles origines. " J'avais fait de l'exil une patrie. " (Semprun, 1994, p. 284)

Une première catégorie d'écrivains bilingues, la plus nombreuse, regroupe ceux et celles qui commencent par écrire dans leur langue maternelle, puis poursuivent leur création dans leur langue seconde. Ainsi, Elsa Triolet a écrit ses premiers romans en russe une fois installée dans son nouveau pays et ne s'est mise à écrire en français qu'en 1938, à la suite du refus de l'URSS de publier son dernier roman (et dans l'espoir de gagner l'approbation 
de Louis Aragon : « J'allais essayer d'écrire en francais pour que tu me dises : écris ! - ou n'écris pas !... en connaissance de cause $\left.{ }^{1} . »\right)$ Parti de son pays à dix-neuf ans, Vladimir Nabokov a écrit huit romans en russe avant d'en écrire huit en anglais. Hector Bianciotti, installé en France depuis les années soixante, y a écrit ses premiers livres en espagnol avant d'adopter le français, pour des raisons linguistiques : " Je sentais bien que mon espagnol, au fil des ans, n'était plus de nulle part, ni d'Espagne ni d'Argentine. Le français était en train de prendre toute la place... " (M. Gazier, 1994)

L'abandon de la langue maternelle pour une autre est souvent très douloureux, voire dramatique. Nabokov, qui connaissait pourtant parfaitement l'anglais depuis l'enfance, se plaint d'avoir " dû " abandonner le russe " my natural language, my natural idiom, my rich, infinitely rich and docile Russian tongue, for a second rate brand of English ${ }^{2}$ ".

À ces écrivains transfuges de la langue maternelle s'oppose un groupe moins nombreux pour qui c'est la langue seconde qui ouvre les sentiers de la création. Ainsi, Samuel Beckett a écrit ses premières auvres en France, mais en anglais, avant de poursuivre une création bilingue. Nancy Huston, anglophone de naissance, a raconté dans Lettres parisiennes - Autopsie de l'exil sa venue à l'écriture grâce au français. Née et élevée dans l'Ouest du Canada, elle est partie vivre aux États-Unis puis, à vingt ans, elle est allée poursuivre ses études en France. C'est là qu'elle s'est fixée, devenant parfaitement bilingue. Elle s'est mise à écrire parce qu'une revue lui avait demandé un texte, et c'est en français qu'elle l'a rédigé, éprouvant, dit-elle un plaisir qu'elle n'aurait « même pas pu imaginer en anglais ", parce que l'anglais avait été « tué par les 'tics' universitaires ", qu'elle n'entendait plus sa langue, que celle-ci l'habitait " comme un poids mort " (L. Sebbar et N. Huston, 1986, p. 97).

1. Cité par Elizabeth Klosty Beaujour (1989), p. 66.

2. Cité par Elizabeth Klosty Beaujour (1989), p. 93. 
Adopter une langue autre, c'est donc échapper aux pesanteurs de son passé linguistique. Elsa Triolet se plaignait d'avoir à lutter contre la tendance des mots à « se remettre dans les mêmes plis »: « On a beau les repasser, les marques restent. [...] Ce que j'appelle les plis, ce sont les tics, les tournures qui vous sont habituelles, si bien que la libre mise en mots devient une mise en plis rigides " (E. Triolet, 1969, p. 98). Roland Barthes disait que " La nausée arrive dès que la liaison de deux mots importants va de soi " (R. Barthes, 1973, p. 70). Changer de langue permet d'échapper à l'éccurement du " ressassant 》 et garantit à Nancy Huston que, grâce à l'usage du français, " pas une seule locution, si galvaudée fưt-elle, n'irait [...] jamais complètement de soi » (L. Sebbar et N. Huston, 1986, p. 158).

Ce n'est qu'à partir du moment où plus rien n'allait de soi ni le vocabulaire, ni la syntaxe, ni surtout le style -, à partir du moment où était aboli le faux naturel de la langue maternelle, que j'ai trouvé des choses à dire. Ma 'venue à l'écriture' est intrinsèquement liée à la langue française. Non pas que je la trouve plus belle ni plus expressive que la langue anglaise, mais étrangère, elle est suffisamment étrange pour stimuler ma curiosité. (Ibid., p. 16)

Quand elle écrit ces lignes, elle a déjà à son actif six livres en français, dont deux textes de fiction.

L'adoption d'une autre langue s'avère ainsi le moyen d'accéder à l'authentique. Dans l'espace que ménage celle-ci du fait même qu'elle est étrangère, elle permet de respirer, elle offre le jeu qui permet l'émergence du JE de l'écriture. Tout récemment, le cas d'Andreï Makine vient de nous en offrir un exemple saisissant. Né au cour de la Russie, il passait ses vacances chez sa grand-mère, une Française échouée dans une petite ville de Sibérie. Cette grandmère lui avait appris le français dès son plus jeune âge en lui racontant les fastes de la Belle Époque et en lui lisant les journaux de sa jeunesse. Cette langue, "mystérieuse matière, invisible et omniprésente ", est la clef qui lui offre l'accès à un autre monde et qui lui donne une autre vision de son propre monde : elle le 
transporte, dans tous les sens du mot. Andrei Makine évoque en ces termes le moment de révélation où il a enfin compris ce qui le différenciait profondément de ses compagnons d'enfance : " Je voyais la Russie en français! J'étais ailleurs. En dehors de ma vie russe. Et ce déchirement était si aigu et en même temps si exaltant que je dus fermer les yeux. " (A. Makine, 1995, p. 51) Le français devient, grâce à l'usage qu'en fait la merveilleuse grand-mère, la " langue d'étonnement " qui le transforme à jamais en habitant de l'ailleurs, et avec laquelle se confond pour lui la littérature. Devenu adulte, il part en France, et c'est en français qu'il trouve les mots pour faire revivre celle qui lui avait laissé entrevoir, dès son enfance, la " mystérieuse consonance des instants éternels " (p. 277), vision très proustienne de la littérature.

Ainsi, c'est la distance, la non-coïncidence avec soi-même, qui permet la création. Nancy Huston rapproche elle aussi langue étrangère et écriture.

N'est-ce pas cette distanciation même qui constitue la littérature ? Notre écriture ne vient-elle pas de ce désir de rendre étranges et étrangers le familier et le familial, plutôt que du fait de vivre, banalement, à l'étranger ? [...] Écrire en français, c'était donc un double éloignement : d'abord écrire, ensuite en français (ou plutôt l'inverse : d'abord en français, ensuite écrire). En d'autres termes, $j$ 'avais besoin de rendre mes pensées deux fois étranges, pour être sûre de ne pas retomber dans l'immédiateté, dans l'expérience brute sur laquelle je n'avais aucune prise. (L. Sebbar et $N$. Huston, 1986, pp. 196-197)

La langue étrangère est doublement libératrice, puisqu'elle libère à la fois de la langue maternelle et du passé avec lequel celle-ci fait bloc. Parlant de ses retours en Amérique anglophone, Nancy Huston dit se sentir étouffée à la fois par sa langue maternelle et par sa mère patrie :

Tout en elles m'étouffe, toutes les nuances de niaiserie depuis les prévisions météorologiques à la radio jusqu'aux conversa- 
tions dans la rue. Je comprends trop bien, ça me colle à la peau : c'est moi - le moi que j'ai fui -, ce sont toutes les platitudes de mon enfance dans les Prairies plates, les mêmes inanités religieuses, les mêmes chansons débiles - et je panique. Là, pour le coup, j'ai le mal du pays, mais comme on dit le mal de mer : mon pays me donne la nausée. (lbid., p. 26)

La langue étrangère est donc ce qui permet de ne pas coïncider avec soi-même, avec pour conséquence, dit Nancy Huston, que son usage provoque le sentiment de " vivre entre guillemets », à distance.

Mais cet écart libérateur peut s'avérer aussi source d'angoisse. L'écrivaine a des cauchemars où elle entend s'épaissir son accent, voit fondre son vocabulaire, se sent perdre le français. " Mon 'vrai moi' transparaît de plus en plus à travers le masque du 'moi' français. Et l'on me condamne à retourner en Alberta. Il s'agit d'une condamnation. Rentrez chez vous, vous n'avez rien à faire ici, vous ne parlez même pas la langue. " (Ibid., p. 181) Comment ne pas voir dans ce cauchemar une anticipation des invectives de Nathalie Petrowski l'accusant d'être « une Albertaine défroqué, une Anglaise récalcitrante qui a renié sa langue maternelle pour épouser le français [...] " (N. Petrowski, 1993, p. D3) ? Passons sur l'incohérence incestueuse de l'image de la journaliste (renier sa mère pour en épouser une autre !) mais reprenons la métaphore des épousailles, car elle se trouve coīncider avec la réflexion d'Elsa Triolet sur son bilinguisme : « On dirait une maladie : je suis atteinte de bilinguisme. Ou encore : je suis bigame. Un crime devant la loi. Des amants, tant qu'on veut : deux maris enregistrés, non. On me regarde de travers : à qui suis-je ? " (E. Triolet, 1969, p. 54) Même image, avec adaptation en fonction du sexe, chez Nabokov, qui écrit à Edmund Wilson : " I have lain with my Russian muse after a long period of adultery and am sending you the big poem she bore $^{3}$. " Cette image récurrente rend bien compte de la culpabilité qui accompagne souvent le passage de l'écrivain à l'autre langue.

3. Cité par Elizabeth Klosty Beaujour (1989), p. 97. 
Toutefois, la répartition des rôles dans la métaphore de l'adultère n'est pas toujours si claire. Comme l'écrivait Elsa Triolet en regard de la statue de femme bifrons qui illustre sa Mise en mots, "Être bilingue, c'est un peu comme d'être bigame : mais quel est celui que je trompe ? " (E. Triolet, 1969, pp. 84-85) (Voir Illustration 1) La symbolisation du bilinguisme par la statue bifrons évoque irrésistiblement la " névrose de Janus ", la "bi-frontalité douloureuse » dont se plaint Claude Esteban (1990, p. 95) dans le très beau livre qu'il consacre à son expérience d'enfant bilingue, $l e$ Partage des mots.

Si Nancy Huston a commencé sa carrière littéraire par un amour " adultère » avec le français, en 1993, elle a renoué avec l'anglais, en laissant ressurgir sa langue et en découvrant son pays d'origine. Doublement déracinée, par le pays et par la langue, d'un même mouvement, elle retrouve les deux :

Avec ce livre [Plainsong], mes racines ont pris de l'intérêt pour moi. J'avais toujours dit à tout le monde que je venais d'un pays plat, avec une histoire inexistante, une culture zéro. Et peu à peu, je me suis aperçue qu'il pouvait y avoir de la passion, de la magie et de la matière littéraire dans mes racines. Et ça m'est venu en anglais. J'entendais la musique de l'anglais. Des cantiques, des chansons de cow-boy, et de travailleurs des chemins de fer. Il fallait que ce soit en anglais. (D. Laurin, 1993)

Le livre est en effet nourri, tissé de ces textes où s'est inscrite la mémoire des Prairies. Le titre anglais, Plainsong, par sa polysémie, qui inclut a la fois le sens de chansons et de cantiques (outre le sens spécifique de " plain-chant»), rend bien cet aspect de l'œuvre.

Ces retrouvailles avec l'anglais ont constitué une expérience " exaltante " pour la romancière parce que, dit-elle, " entre temps, l'anglais était presque devenu langue étrangère pour moi, et c'était analogue à ce qui s'était passé il y a 20 ans quand j'avais commencé à écrire en français, langue étrangère " (C. Klein-Lataud, 1993). Pour reprendre l'interprétation allégorique de la statue d'Elsa 
Pawr les bilingues se traduire desratt Etre facile? trak pas? ou st regarde chame dans use glace, on $I^{\prime} y$ chatede, ne reexfenait pas sor te-

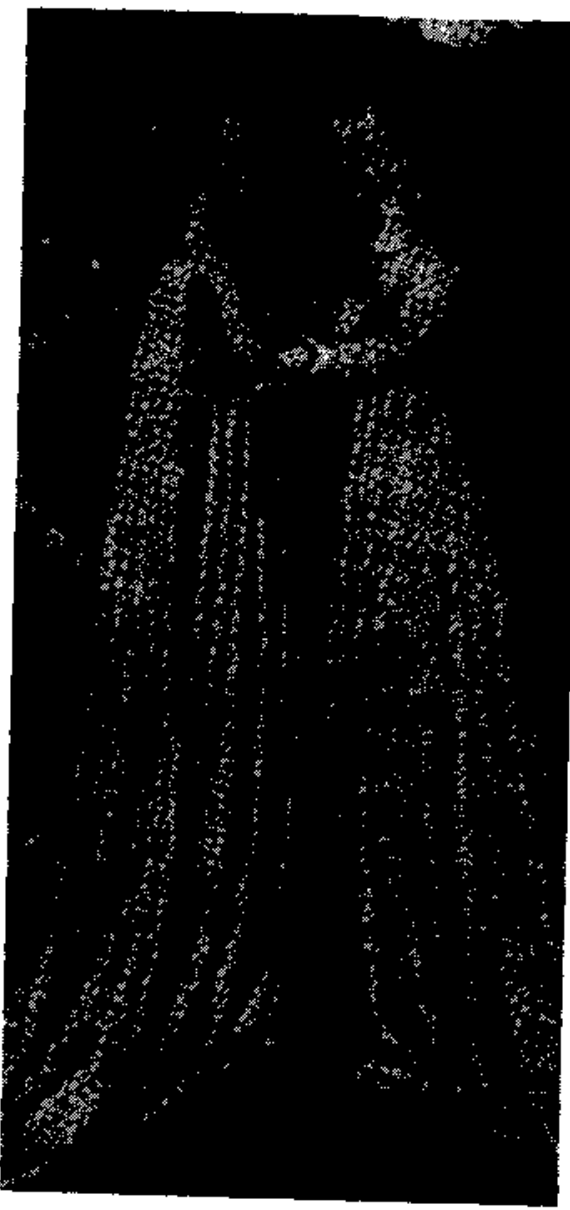

Illustration 1 
Triolet, c'est l'autre visage que l'écrivaine regarde dans le miroir, et elle le découvre d'un cil neuf.

Le choix de la langue dans laquelle va s'opérer la " mise en mots » de l'œuvre est déterminant. Nous en trouvons un témoignage dans le Langage et son double, de Julien Green. On sait que cet écrivain, né à Paris de parents américains, a le français comme langue d'expression artistique. Réfugié aux États-Unis au début de la Seconde Guerre mondiale, il décide d'écrire un livre en hommage à la France, et le commence, comme tout le reste de son ceuvre, en français. Puis il prend conscience de l'impossibilité de se faire publier en français aux États-Unis, et recommence derechef en anglais, avec l'intention de reprendre ce qu'il avait déjà écrit. Or, dit-il,

l'inattendu arriva. Sachant très bien ce que je voulais dire, je commençai mon livre, écrivis une page et demie, mais en me relisant, je m'aperçus que j'écrivais un autre livre, d'un ton si complètement différent du texte français que tout l'éclairagedu sujet était transformé. En anglais, j'étais devenu quelqu'un d'autre. Je continuai. De nouveaux trains de pensées démarrèrent dans mon esprit, de nouveaux convois d'idées se formèrent. La ressemblance entre ce que j'écrivais maintenant en anglais et ce que j'avais écrit en français était si petite qu'on aurait pu douter que ce fût du même auteur. (J. Green, 1987, p. 175)

$\mathrm{JE}$ est un autre, on le savait, mais la différence de langue favorise cette multiplication. Pour parler des différences provoquées chez lui par l'utilisation d'une langue, Nabokov emploie l'expression de " réincarnation ». Julien Green parle de " renaissance " :

Le désir de s'exprimer doit insuffler [à l'écrivain qui change de langue] l'élan de franchir tous les obstacles, de renaître en quelque sorte dans une autre langue, de se faire adopter, de donner à l'inconnu au fond de lui-même les chances de l'aventure humaine. (p. 159) 
La couverture du texte bilingue porte comme nom d'auteur Julian Green, avec en dessous, en petits caractères, la mention « traduit par Julien Green ». Julian, Julien, l'onomastique nous renvoie elle aussi à l'image de l'être double. Les deux versions linguistiques, disposées en regard sur les pages de l'édition bilingue, ne se correspondent d'ailleurs pas totalement ; ainsi, l'extrait que je viens de citer constitue un ajout par rapport au texte anglais dont il est la traduction...

Le Langage et son double nous permet d'aborder la question de l'auto-traduction, exercice périlleux, qui s'est avéré tantôt douloureux, tantôt épuisant pour la plupart de ceux et celles qui s'y sont livrés. La légende d'une gravure choisie par Elsa Triolet pour la Mise en mots nous livre le sentiment éprouvé généralement par les auteurs auto-traducteurs : " Pour les bilingues se traduire devrait être facile ? Non pas ! On se regarde dans une glace, on s'y cherche, ne reconnaît pas son reflet. 》 (E. Triolet, 1969, p. 76) (Voir Illustration 2)

Pourquoi s'autotraduire? L'écrivain le fait parfois parce qu'il a été déçu par les traductions de ses cuvres, comme Nabokov, qui déplore d'y être contraint et trouve une métaphore barbare pour évoquer cette activité : " a terrible thing, sorting through one's own innards, and then trying them on for size like a pair of gloves ${ }^{4}$." L'auto-traduction peut aussi découler d'une position de principe. Ainsi Nancy Huston, commentant sa " réćcriture " de Plainsong en français, explique : " Je n'aurais fait confiance à personne pour le traduire. Quand la première version a été terminée, je l'ai réćcrite en français. " (D. Laurin, 1993) L'auteure insiste sur le terme de récriture, pour exclure la notion de " traduction ". Il y a des raisons pragmatiques à ce choix. On connaît, en effet, la polémique déclenchée en 1993 par l'attribution à Cantique des plaines du Prix

4. Cité par Elizabeth Klosty Beaujour (1989), p. 90 : « une chose abominable, fouiller ses propres entrailles, puis les essayer comme une paire de gants pour trouver la bonne taille " (notre trad.). 


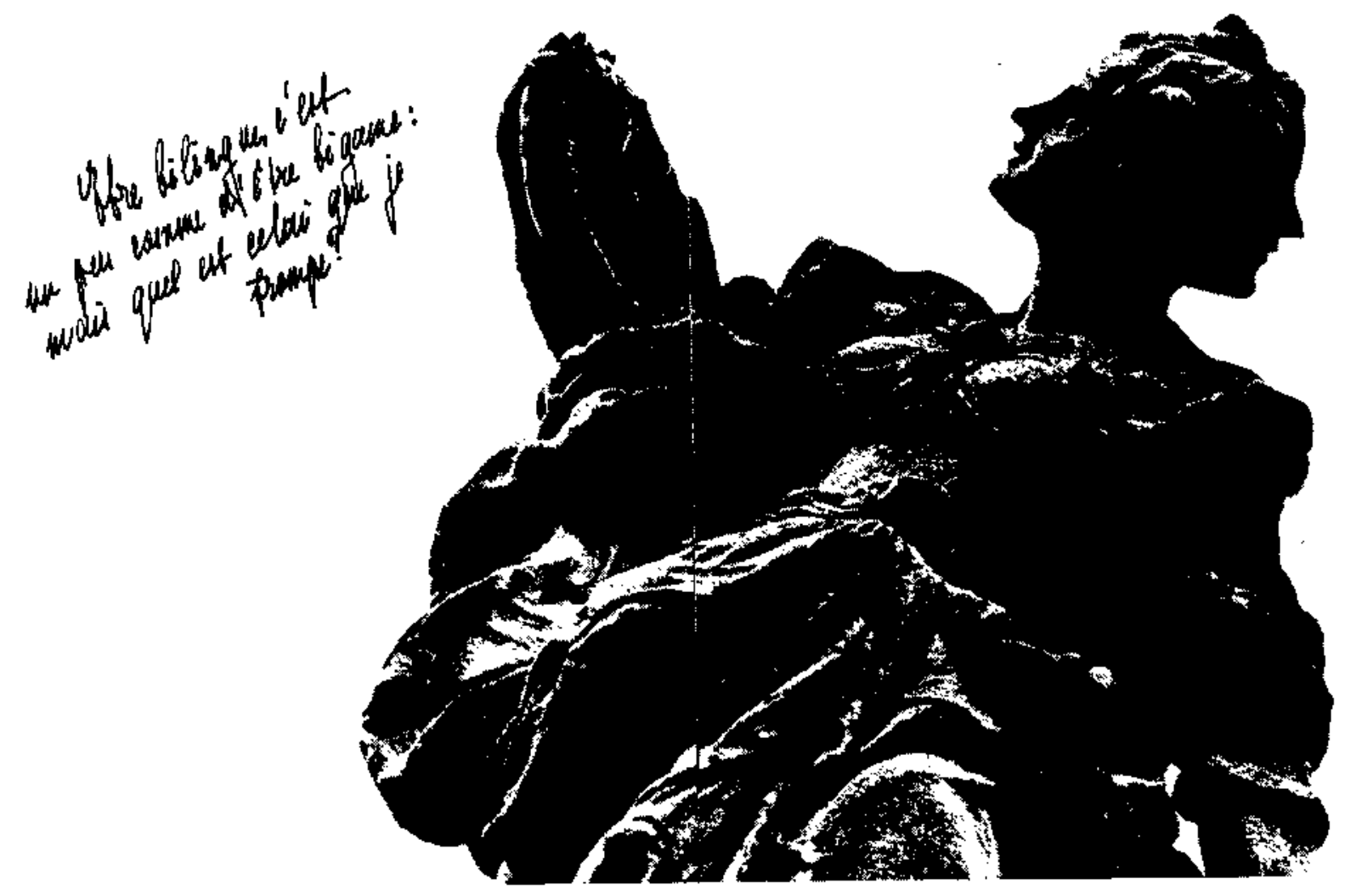

Illustration 2 
du Gouverneur général dans la catégorie « Romans et nouvelles " en français'. L'intervention de cinq éditeurs montréalais auprès du Conseil des Arts pour demander l'annulation de la décision du jury reposait sur la nature du texte, jugé par eux admissible exclusivement dans la catégorie "Traductions ". Pour battre en brèche cet argument, Nancy Huston a donc présenté son livre comme une re-création. Mais toute traduction n'est-elle pas recréation ? L'examen des deux textes Plainsong et Cantique des plaines n'interdit nullement d'utiliser le terme traduction pour désigner le second. En abordant leur comparaison, je m'attendais à les voir diverger, comme les versions bilingues de Julian-Julien Green, ou comme les textes de Nabokov, champion tourmenté des auto-traductions. Citons ses mémoires d'enfance et de jeunesse, rédigés en anglais, " traduits " en russe par lui-même, avec de très importants remaniements, qui aboutirent à un texte si différent de l'original que l'auteur re-traduisit en anglais sa " traduction 》 russe... (Le va-et-vient aurait pu continuer indéfiniment, dialogue sans doute entre le Russe et l'Américain. Et dire que Nabokov aurait pu être aussi, pour peu qu'il l'eût voulu, un grand écrivain de langue française...)

Jorge Semprun, dont j'ai évoqué plus haut le choix du français comme langue de création pour l'Écriture ou la Vie raconte son émotion en se voyant offrir une édition espagnole de cette cuvre dont toutes les pages étaient vierges, puisque le livre avait été interdit par la censure espagnole. Il s'était dit alors qu'il réécrirait ce livre sur les pages blanches de l'exemplaire unique, en espagnol, et " sans tenir compte de la traduction existante " (traduction en espagnol publiée au Mexique). Son ami l'écrivain Carlos Fuentes lui fait alors remarquer qu'il aurait da écrire lui-même la version espagnole, et ajoute ces réflexions intéressantes:

Tu n'aurais pas simplement traduit, tu aurais pu te permettre de te trahir. De trahir ton texte originaire pour essayer d'aller plus loin. Du coup, un livre different aurait surgi, dont tu

5. Pour les détails de cette affaire, voir Robert Yergeau (1994). 
aurais pu faire une nouvelle version française, un nouveau livre. (J. Semprun, 1994, pp. 284-285)

Il aurait ainsi réalisé, en cheminant d'une langue à l'autre, le rêve que Carlos Fuentes attribue à tout écrivain : passer sa vie à écrire un seul livre, sans cesse renouvelé !

$\mathrm{Au}$ contraire, les versions anglaise et française du roman de Nancy Huston sont tout à fait parallèles. À l'affût de différences signifiantes, je m'étais interrogée sur la différence des épigraphes, le roman anglais ayant une citation de Flannery O'Connor : "Then you ain't saved? ", le roman français s'ouvrant sur une ligne de Lennon et McCartney : "No one you can save that can't be saved. "S'agissait-il d'un choix opéré en fonction du lectorat, les Beatles étant choisis pour les Français parce qu'ils sont universellement connus, alors que Flannery $O^{\prime}$ Connor était présumée référence trop obscure ? Pas du tout. La cáuse de cette différence est purement juridique : si les Beatles ne figurent pas dans l'édition anglaise, c'est que les ayants droit n'ont pas donné l'autorisation de reproduire la phrase pour l'édition anglaise ${ }^{6}$ !

Le texte proprement dit comporte une seule suppression importante, celle d'un passage où l'anniversaire de la Confédération canadienne est l'occasion d'un commentaire politique et d'une comparaison entre les autochtones canadiens et les Algériens, qui ont conquis leur indépendance et expulsé les Français de leur pays (les pages 197-198 de Plainsong correspondant à la page 238 du Cantique des plaines). Quant au reste, les textes ne different que dans la mesure où toute traduction diffère obligatoirement du texte source, en particulier par l'effet d'hétérogénéité des noms propres et des références. Ainsi, dans la version anglaise, les chansons et les hymnes sont tissés dans le texte avec lequel ils forment un tout thématique et linguistique, alors que dans la version française, cités généralement en anglais et suivis de leur traduction, ils tranchent.

6. Nancy Huston, Lettre à Christine Klein-Lataud, 15 mai 1995. 
Les différences stylistiques sont, quant à elles, plus difficiles à analyser. La syntaxe des deux textes est analogue, caractérisée par de longues phrases méandreuses, s'étirant parfois à l'infini, comme les Prairies qu'ils évoquent, comme l'histoire des peuples et des individus. La différence la plus notable que j'aie relevée est celle des registres. Souvent, là où l'anglais est standard, le français est familier ou argotique. Voici quelques exemples : "I tell you I'm miserable/ je te dis que je suis en train de crever." "God [...] roared with laughter/ Dieu [...] se fendant la gueule. " "The kids just died and died/ Les enfants crevaient. " " God's word/ le baratin de Dieu. " " Can you believe it ?/ Est-ce foutrement croyable ? " Il arrive parfois qu'un imparfait du subjonctif vienne "littérariser " le texte français, mais le mouvement général se fait vers l'argot. Cela pose un problème au lectorat francophone d'Amérique parce que, comme on le sait, c'est dans ce registre qu'il y a le plus d'écart entre les variétés régionales, et que l'argot parisien détonne parfois dans le contexte canadien qui est celui du livre. Ainsi les adjectifs décrivant l'archétype de pionnière, " tall and tough and healthy " sont rendus par " balèze et baraquée ", mots qui n'appartiennentpas au vocabulaire d'ici, d'où l'impression d'une fausse note.

Il faut toutefois remarquer que ce choix, outre qu'il est naturel pour une écrivaine parisienne de fait, s'appuie sur des raisons phonologiques, et que Nancy Huston a répété que ses choix s'opéraient en fonction de la « musique " des phrases :

le rythme, la phonétique, c'est-à-dire la musique en général, pour moi, sont primordiales. Donc souvent, j'ai été prête à sacrifier le sens précis des mots pour preserver un certain nombre de syllabes, ou pour préserver une allitération. (C. Klein-Lataud, 1993)

L'exemple le plus spectaculaire de cette stratégie nous est fourni par le passage suivant, qui raconte l'expropriation des Indiens par le CPR. Les Blancs assurent la déchéance du chef Crowfoot en lui tenant figurativement ce discours : " Hove a drink. Have another drink. All right, see? Hit the gutter. Oh give me a home where the buffalo roam." (N. Huston, 1993a, p. 50) En voici la traduction : 
le même rayonnement ; depuis cette époque, les amonomin жigneurs Jacaux se sont approprié tant de moulins et de forges, tant de champs et de vignes ecclésiastiques, qu'en ce moment la situation financière du puthest plus que precaire ; la prieure Marie de Lagranize vient de dresser un pitoyable bilan des revenus et des dépenses, et d'en tiker la conclusion que décidément celles-ci dépassent a dew aw j

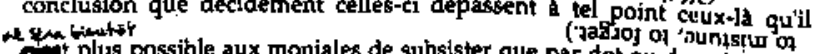

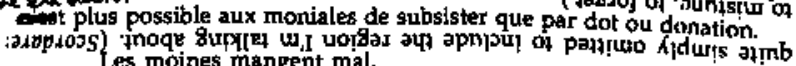

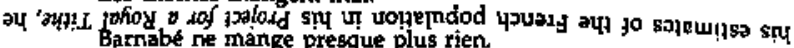

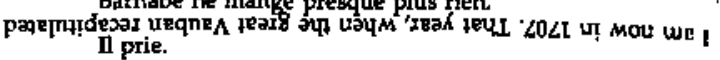

"seop Kfurptins i]

Le bon Père Thomas tient sa promesse; le soir mème du décí

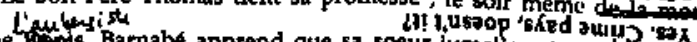

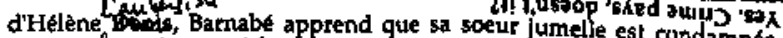
britie area la weace yusligur. incente Entend toute l'affreuse histoire, avec tous ses affreux détails.

I passe la nuik à pleurer Barbe, et as se remémin - אdduct ure I

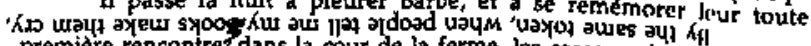
premiere rencontref dans la cour de la ferme, les caresses, les $h_{f}$

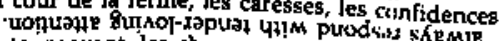
- et puis tout à coup, deja, toumoyant, les chauve-souris. C'est ainsi. Sur

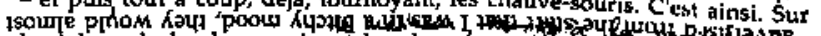
les talons du bonheut arrive tes-

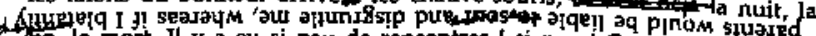

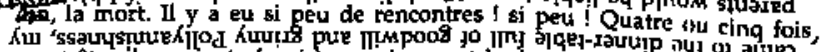
peut-étre ils,avaient réussi a se voir, avant la danse n....min dew fiux de la

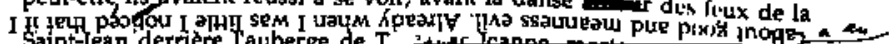

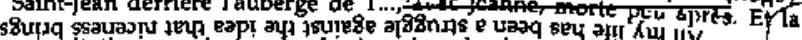
consultation angoissee, clandestine, dans la chapelte dy

avait pris la fuite. Puis plus rien, pendant si longtermps rien.. Jit mainte-

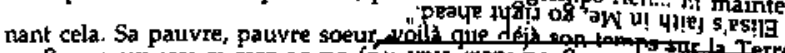

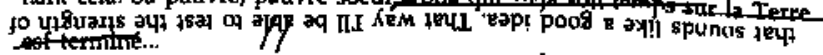

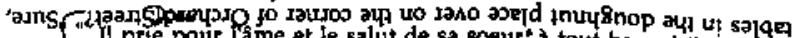
Il prie pour jame et le salut de sa soeur: a tout hasard il demande

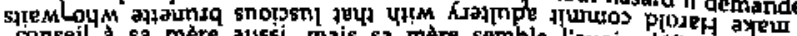
conseil à sa mère aussi, mais sa mère semble l'avoir définjitivement

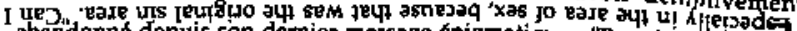
abandorné depuis son dernier message énigmatique : "Prends garde !" 
« Bois encore un coup. Encore un petit coup. Vous voyez bien comme tout va bien! Et maintenant, la guitare ! Oh give me a home Where the buffalo roam. " (N. Huston, 1993b, p. 67) "Gutter 》 et " guitare " sont des paronymes, mais leurs signifiés, bien sûr, n'ont rien de commun, quoique les deux sens s'inscrivent chacun parfaitement dans le texte, Hit the gutter reprenant parodiquement Hit the road, thème de la ruée vers l'ouest, et " guitare " introduisant logiquement la chanson qui suit. C'est le seul passage où Nancy Huston m'ait paru excéder les « droits " du traducteur et user de la liberté sans bornes de l'auteur.

Si les deux textes sont si proches, c'est aussi à cause de la façon dont ils ont été écrits. En effet, Nancy Huston a raconté qu'elle les a élaborés parallèlement :

C'était fascinant, il y avait un aller et retour pendant plus d'un an entre les deux langues, parce que la "traduction ", ça oblige toujours à voir quelles sont les faiblesses du texte original. Donc, grâce au français, $\mathrm{j}$ 'améliorais l'anglais et viceversa. [...] C'était assez fastidieux, c'était très long, c'était un peu comme traîner des pierres. (C. Klein-Lataud, 1993)

Tout récemment, Robert Lepage soulignait le même effet rétroactif de la traduction sur le texte originel. "Translating your own work forces you to go deeper into meaning ", a-t-il dit (K. Taylor, 1995, p. C1.2), en expliquant qu'il a réécrit sa version française à partir du travail qu'il avait fait pour la traduire en anglais. Nancy Huston continue à travailler l'entre-deux puisqu'elle vient de terminer un roman, Instruments des ténèbres (1996), qu'elle a écrit à moitié en anglais, à moitié en français. Le livre se présente comme le carnet de bord tenu par une romancière new-yorkaise, qui raconte sa vie en anglais tout en écrivant en français un roman situé dans le Berry au début du XVII'` siècle. Les deux textes, lus en alternance, laissent peu à peu apparaître des ressemblances profondes. La page de brouillon ci-contre, authentique (voir Illustration 3), témoigne de la juxtaposition hallucinante des deux langues, qui vous met littéralement la tête à l'envers. Bien sûr, le texte ne sera pas publié sous cette forme ; il n'exigera même pas des lecteurs qu'ils soient 
bilingues : Nancy Huston a traduit la moitié anglaise en français (et vice-versa pour une éventuelle publication en anglais). Mais le manuscrit est comme la représentation symbolique du travail des langues côte à côte.

Ces expériences remettent en question la notion d' " original ", quand l'auteur des deux textes est le même. Elizabeth Beaujour cite le cas de Raymond Federman, né à Paris et venu vivre aux États-Unis à dix-neuf ans, qui travaille conjointement sur les versions anglaise et française de ses textes, et qui a publié un texte bilingue, "D'une parenthèse à l'autre ", " From One Parenthesis to Another ", où la version anglaise dit « traduit du français par l'auteur » et la version française « traduit de l'anglais par l'auteur » (E. K. Beaujour, 1989, p. 199). Beaucoup d'écrivains ne soutiennent-ils pas, d'ailleurs, que tout texte est la traduction d'un texte idéal n'existant que dans l'esprit de son créateur, qui ensuite en opère la " mise en mots » dans une ou plusieurs langues? Agnès Whitfield a précisé "Traduction sans original " en tête de son recueil poétique $O$ cher Émile je t'aime, et Jacques Roubaud confie : "En fait, je ne suis dans aucune langue, toujours traduisant?." Régine Robin commente ainsi ce sentiment, pour en faire le propre de l'écriture :

Impossible pour l'écrivain de se situer tout à fait dans sa ou ses langues, de faire corps avec sa langue natale ou maternelle, d'habiter complètement son nom propre ou sa propre identité, impossible de coinncider avec soj-même ou avec un quelconque fantasme d'unité du sujet, impossible peut-être même d'occuper une place de sujet autrement que dans l'écriture. (p. 9)

Le bilinguisme, qui apparaît de prime abord comme un surcroît de richesse, peut aussi s'avérer, nous l'avons vu, source de malaise. Elsa Triolet a écrit : « Ainsi, moi, je suis bilingue. Je peux traduire ma pensée également en deux langues. Comme conséquen-

7. Cité par Régine Robin (1994), p. 9. 
malaise. Elsa Triolet a écrit : « Ainsi, moi, je suis bilingue. Je peux traduire ma pensée également en deux langues. Comme conséquence, j'ai un bi-destin. Ou un demi-destin. Un destin traduit. " (E. Triolet, 1969, p. 8) Et Nancy Huston utilise une image analogue : " au bout de dix années de vie à l'étranger, loin d'être devenue 'parfaitementbilingue', je me sens doublement mi-lingue. ") (L. Sebbar et N. Huston, 1986, p. 74) L'auto-traduction ou la création parallèle peuvent alors apparaître comme une façon de transcender le clivage, de réconcilier les deux moitiés de l'être intérieurement déchiré en faisant cohabiter harmonieusement les deux langues. Elizabeth Klosty Beaujour conclut son étude des écrivains bilingues sur le triomphe de l'auto-traduction :

a successful self-translation is [...] the ultimate triumph. It provides the Mephistophelian pleasure of creating two mutually orbiting works in dynamic equilibrium [...]. In a successful self-translation, the writer finds alternatives and compensations in the two linguistic systems at his disposal, and the text passes. The voice passes, and is unmistakably the same in both languages, and this very fact indicates that it emanates from a self which must exist below both languages, flowing up through the growth rings of the tree, manifesting itself in a bifurcating trunk and in separate systems of branches and leaves, all of which are in active balance ${ }^{8}$.

8. E. K. Beaujour (1989), pp. 175, 176 : « une auto-traduction réussie représente le succès suprême. Elle procure le plaisir méphistophélique de créer deux mondes en orbite l'un par rapport à l'autre dans un équilibre dynamique [...]. Dans une auto-traduction réussie, l'écrivain trouve des solutions et des compensations entre les deux systèmes linguistiques dont il dispose, et le texte passe. La voix passe, et on la retrouve identique dans les deux langues; ce fait suffit à montrer qu'elle émane d'un moi qui doit exister en dessous des deux langues, qui remonte comme la sève à travers les anneaux de croissance de l'arbre, donne naissance à un tronc fourchu et à deux systèmes distincts de branches et de feuilles, tous poussant de façon équilibrée " (notre trad.). 
L'image de l'arbre nous ramène au thème d'une Babel heureuse, et pour terminer sur cette note euphorique, j'emprunterai ma conclusion à Roland Barthes : " le vieux mythe biblique se retoume, la confusion des langues n'est plus une punition, le sujet accède à la jouissance par la cohabitation des langages, qui travaillent côte à côte : le texte de plaisir, c'est Babel heureuse. » (p. 10)

\section{Références}

BARTHES, Roland (1973). Le Plaisir du texte. Paris, Éd, du Seuil.

BEAUJOUR, Elizabeth Klosty (1989). Alien Tongues - Bilingual Writers of the 'First' Emigration. Ithaca et Londres, Cornell University Press.

ESTEBAN, Claude (1990). Le Partage des mots. Paris, Gallimard, coll. « L'un et l'autre ».

GAZIER, Michèle (1994). Interview d'Hector Bianciotti, Télérama, $n^{\circ} 2306,26$ mars.

GREEN, Julian (1987). « Une expérience en anglais ", Le Langage et son double. Paris, Éd. du Seuil, coll. « Points ».

HUSTON, Nancy (1993a). Plainsong. Toronto, Harper-Collins.

Leméac/Actes Sud.

(1993b). Cantiques des plaines. Montréal/Paris, (1996). Instruments des ténèbres. Montréal/Paris,

Leméac/Actes Sud.

KLEIN-LATAUD, Christine (1993). Interview de Nancy Huston, Société Radio-Canada, 16 novembre. 
LAURIN, Danielle (1993). « Source sûre », interview de Nancy Huston, Voir, 16-22 septembre.

MAKINE, Andrei (1995). Le Testament français. Paris, Mercure de France.

PETROWSKI, Nathalie (1993). « Bar Payant », La Presse, 18 nov., p. D3.

ROBIN, Régine (1994). Le Deuil de l'origine. Une langue en trop, la langue en moins. Paris, Presses universitaires de Vincennes.

SEBBAR, Leilla et Nancy HUSTON (1986). Lettres parisiennes Autopsie de l'exil. Paris, B. Barrault.

SEMPRUN, Jorge (1994). L'Écriture ou la Vie. Paris, Gallimard, coll. " NRF ».

TAYLOR, Kate (1995). « The non-stop Robert Lepage », The Globe and Mail, 17 mai, p. Cl.2.

TRIOLET, Elsa (1969). La Mise en mots. Genève, Skira, coll. « Les Sentiers de la création $»$.

WHITFIELD, Agnès (1993). O cher Émile je t'aime. Hearst, Éditions du Nordir.

YERGEAU, Robert (1994). À tout prix. Les prix littéraires au Québec. Montréal, Éd. Triptyque, ch. 4.

RÉSUMÉ : Les voix parallèles de Nancy Huston - Cet article s'attache à la problématique des écrivains bilingues ou polyglottes (Elsa Triolet, Vladimir Nabokov, Julien Green), et plus particulièrement à celle de Nancy Huston. Quelles raisons poussent un écrivain ou une écrivaine à utiliser pour la création une langue seconde plutôt que sa langue maternelle? Quand l'auteur s'auto-traduit, s'agit-il d'une nouvelle création ? Nancy Huston était-elle en droit de 
recevoir le Prix du gouverneur général dans la catégorie fiction fancophone pour Cantique des plaines, "réecriture " de Plainsong par l'auteure?

ABSTRACT : Nancy Huston's Parallel Voices - This article examines the case of bilingual and polyglot writers, such as Nancy Huston, Elsa Triolet, Vladimir Nabokov and Julian Green. Why does a writer choose to write in his or her second language ? When an author translates his or her own work, is this a new text ? Was Nancy Huston eligible to receive the Governor-General's Award in the French fiction category for Cantique des plaines, her own rewriting of her novel Plainsong ?

Christine Klein-Lataud : École de traduction, Collège universitaire Glendon, Université York, 2275, av. Bayview, Toronto, Ontario, M4N 3M6, CANADA 